\title{
Sufficient descent three term conjugate gradient method via symmetric rank-one update for large-scale optimization
}

\begin{abstract}
In this paper, we propose a three-term conjugate gradient method via the symmetric rank-one update. The basic idea is to exploit the good properties of the SR1 update in providing quality Hessian approximations to construct a conjugate gradient line search direction without the storage of matrices and possess the sufficient descent property. Numerical experiments on a set of standard unconstrained optimization problems showed that the proposed method is superior to many well-known conjugate gradient methods in terms of efficiency and robustness.
\end{abstract}

Keyword: Unconstrained optimization; Symmetric rank-one update; Three-term conjugate gradient method; Memoryless update; Sufficient descent 